Sādhanä, Vol. 18, Part 5, September 1993, pp. 869-889. (C) Printed in India.

\title{
Analytical formulation of small signal stability analysis of power systems with nonlinear load models
}

\author{
RAJEEV K RANJAN, M A PAI and P W SAUER \\ University of Illinois, Department of Electrical and Computer Engineering, \\ Urbana, IL 61801, USA
}

\begin{abstract}
It is now recognized that both load dynamics and generating unit dynamics contribute significantly to the limitation of loadability of power systems. In this paper, we take a static nonlinear load representation and different combinations of machine and exciter dynamic models to develop a comprehensive linearized model to study small signal stability. In particular we monitor the Hopf bifurcation instability and through participation factor analysis we identify the relevant state variables.
\end{abstract}

Keywords. Small signal stability; Hopf bifurcation; nonlinear load models; power system stability.

\section{Introduction}

There is extensive literature on small signal analysis in power systems (Padiyar et al 1981; Kundur et al 1990; Sauer \& Pai 1990; Wang \& Semlyen 1990), and it is the principal tool to study both low-frequency oscillations and voltage stability. In the literature these two phenomena are treated separately. In this paper, we take a unified approach and attempt to look at the dependence of these two phenomena on the parameters of the system, which are the loads (nonlinear dependence on voltage) and modelling of the generating unit (machine and exciter). Use of participation factor is helpful in identifying state variable participation in a critical mode. We also extend the results of an earlier paper (Sauer \& Pai 1990) regarding the role of the system Jacobian, algebraic Jacobian and the load flow Jacobian on voltage collapse. To illustrate our studies both 3-machine and 10-machine cases are studied but the research grade program developed is general enough to handle larger systems.

The small-perturbation behaviour of the power system in the vicinity of a steadystate operating point can be described to first order by a set of linear, time-invariant (LTI) differential equations in the state space form

$$
\dot{x}=A x+B u \text {. }
$$

The $N$-dimensional state vector $x$ represents the perturbations of the system state variables from their nominal values at the given operating condition, and the vector $u$ represents perturbations of the system inputs such as voltage reference, desired real power or load demands. The numerical values of the matrices $A$ and $B$ depend on 


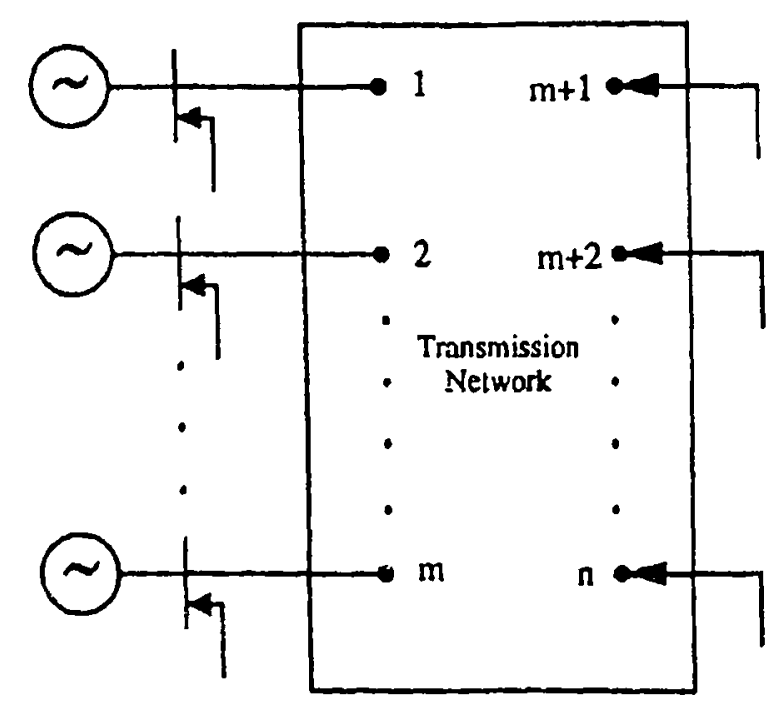

Figure 1. A general $m$-machine, $n$-bus system.

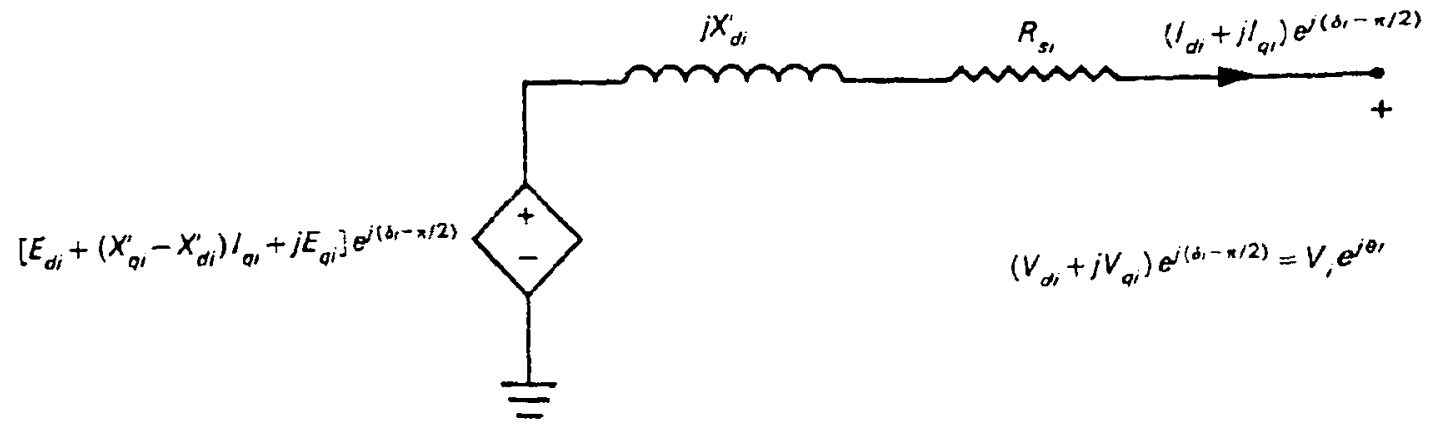

Figure 2. Synchronous machine two-axis model dynamic circuit $(i=1, \ldots, m)$.

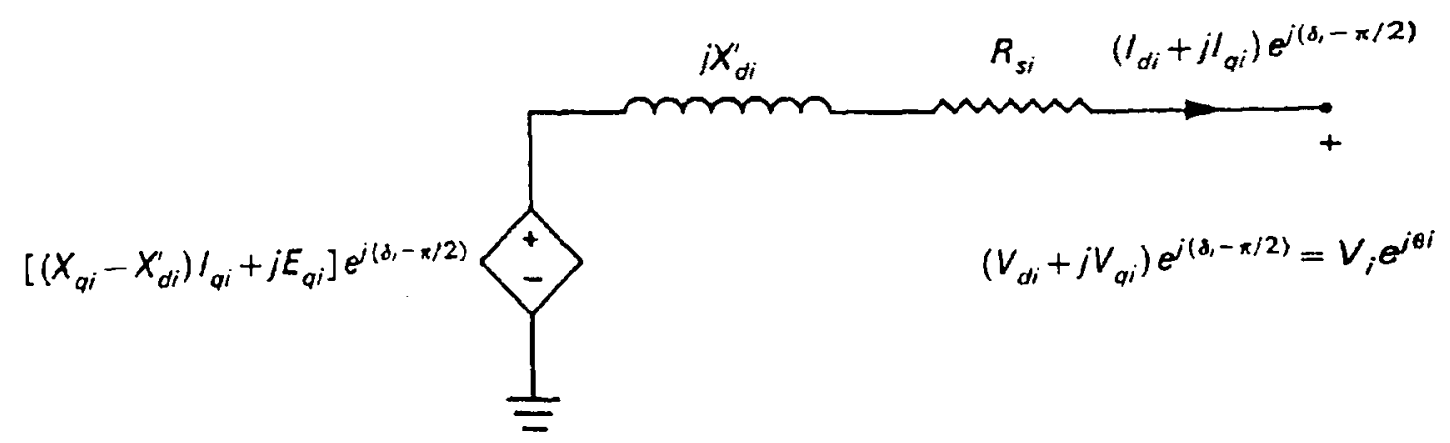

Figure 3. Synchronous machine flux-decay model dynamic circuit $(i=1, \ldots, m)$.

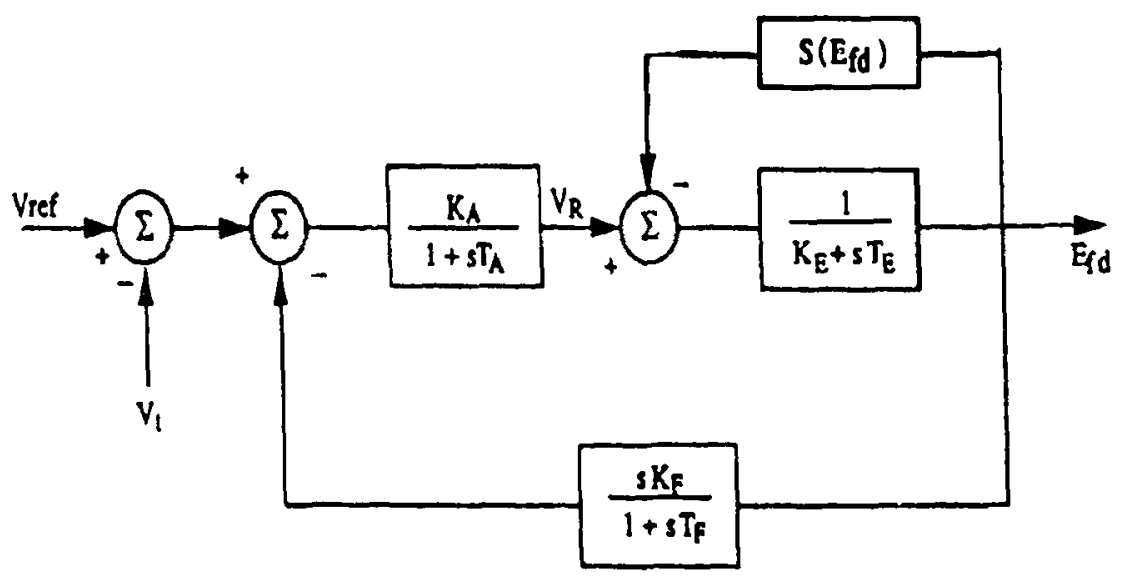

Figure 4. IEEE-type 1 exciter model. 


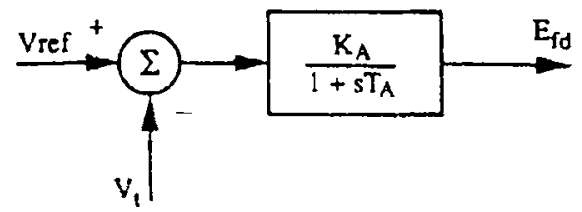

Figure 5. Static exciter model,

the operating condition as well as on the system parameters. The whole analysis starts with a systematic derivation of a linear model for an $n$-bus $m$-machine nonlinear differential algebraic system with nonlinear voltage-dependent loads at the network buses (figure 1). The model so obtained is flexible enough to study both low-frequency oscillations and voltage stability problems. For the former study the system $A$ matrix and its eigenvalues are readily obtainable for any given load model. The influence of parameters such as exciter gain and loads can be studied very easily. It is shown that the appearance of the electromechanical mode of oscillation or exciter mode oscillation depends critically on the modelling of the machine and the excitation system. For voltage stability analysis, we progressively load the system at a bus or set of buses and, at each loading, monitor the eigenvalues of the linearized system.

The machine is modelled by either a two-axis or a flux decay model, and the excitation system by either an IEEE-type I or a static exciter model. Thus potentially we have four types of generating unit models. We derive two linearized models, namely, a two-axis, with IEEE-type 1 exciter (model A) or flux decay with static exciter (model B). The synchronous machine representation and the exciter representations are shown in figures $2-5$. One could also consider a two-axis machine model with a static exciter (model $\mathrm{C}$ ) and a flux decay model of the machine with IEEE-type 1 exciter (model D).

\section{Various mathematical models}

The mathematical model consists of differential equations pertaining to machine and exciter dynamics and algebraic equations corresponding to the stator and network equations.

\subsection{Model A (two-axis model with IEEE-type 1 exciter)}

The differential equations of the machine and the exciter are given as in Sauer \& Pai (1991) where the various symbols are defined.

\section{1a Differential equations:}

$$
\begin{aligned}
\frac{\mathrm{d} \delta_{i}}{\mathrm{~d} t} & =\omega_{\mathrm{i}}-\omega_{s}, \\
\frac{\mathrm{d} \omega_{i}}{\mathrm{~d} t} & =\frac{T_{M i}}{M_{i}}-\frac{\left[E_{q i}^{\prime}-X_{d i}^{\prime} I_{d i}\right] I_{q l}}{M_{i}}-\frac{\left[E_{d i}^{\prime}+X_{q i}^{\prime} I_{q i}\right] I_{d i}}{M_{i}}-\frac{D_{i}\left(\omega_{i}-\omega_{s}\right)}{M_{i}}, \\
\frac{\mathrm{d} E_{q i}^{\prime}}{\mathrm{d} t} & =-\frac{E_{q i}^{\prime}}{T_{d o i}^{\prime}}-\frac{\left(X_{d i}-X_{d i}^{\prime}\right) I_{d i}}{T_{d o i}^{\prime}}+\frac{E_{f d i}}{T_{d o i}^{\prime}} \\
\frac{\mathrm{d} E_{d i}^{\prime}}{\mathrm{d} t} & =-\frac{E_{d i}^{\prime}}{T_{q o i}^{\prime}}+\frac{I_{q i}}{T_{q o i}^{\prime}}\left(X_{q i}-X_{q i}^{\prime}\right)
\end{aligned}
$$




$$
\begin{aligned}
\frac{\mathrm{d} E_{f d i}}{\mathrm{~d} t} & =-\frac{K_{E i}+S_{E}\left(E_{f d i}\right)}{T_{E i}} E_{f d i}+\frac{V_{R i}}{T_{E i}}, \\
\frac{\mathrm{d} V_{R i}}{\mathrm{~d} t} & =-\frac{V_{R i}}{T_{A i}}+\frac{K_{A i}}{T_{A i}} R_{f i}-\frac{K_{A i} K_{F i}}{T_{A i} T_{F i}} E_{f d i}+\frac{K_{A i}}{T_{A i}}\left(V_{r e f i}-V_{i}\right), \\
\frac{\mathrm{d} R_{F i}}{\mathrm{~d} t} & =-\frac{R_{F i}}{T_{F i}}+\frac{K_{F i}}{\left(T_{F i}\right)^{2}} E_{f d i}, \quad \text { for } i=1, \ldots, m .
\end{aligned}
$$

2.1b Stator algebraic equations: The stator algebraic equations are

$$
\begin{aligned}
& E_{d i}^{\prime}-V_{i} \sin \left(\delta_{i}-\theta_{i}\right)-R_{s i} I_{d i}+X_{q i}^{\prime} I_{q i}=0, \\
& E_{q i}^{\prime}-V_{i} \cos \left(\delta_{i}-\theta_{i}\right)-R_{s i} I_{q i}-X_{d i}^{\prime} I_{d i}=0, \text { for } i=1, \ldots, m .
\end{aligned}
$$

These algebraic equations can be represented as a current dependent voltage source at the generator buses (figure 2).

2.1c Network equations: The network equations are

$$
\begin{aligned}
& I_{d i} V_{i} \sin \left(\delta_{i}-\theta_{i}\right)+I_{q i} V_{i} \cos \left(\delta_{i}-\theta_{i}\right)+P_{L i}\left(V_{i}\right) \\
& \quad-\sum_{k=1}^{n} V_{i} V_{k} Y_{i k} \cos \left(\theta_{i}-\theta_{k}-\alpha_{i k}\right)=0, \\
& \begin{aligned}
I_{d i} V_{i} \cos \left(\delta_{i}\right. & \left.-\theta_{i}\right)-I_{q i} V_{i} \sin \left(\delta_{i}-\theta_{i}\right)+Q_{L i}\left(V_{i}\right) \\
& \quad-\sum_{k=1}^{n} V_{i} V_{k} Y_{i k} \sin \left(\theta_{i}-\theta_{k}-\alpha_{i k}\right)=0, \quad i=1, \ldots, m,
\end{aligned} \\
& P_{L i}\left(V_{i}\right)-\sum_{k=1}^{n} V_{i} V_{k} Y_{i k} \cos \left(\theta_{i}-\theta_{k}-\alpha_{i k}\right)=0, \\
& Q_{L i}\left(V_{i}\right)-\sum_{k=1}^{n} V_{i} V_{k} Y_{i k} \sin \left(\theta_{i}-\theta_{k}-\alpha_{i k}\right)=0, \text { for } i=m+1, \ldots, n .
\end{aligned}
$$

\subsection{Model B (flux decay model and fast exciter)}

If the damper winding time constants $T_{q o i}^{\prime}$ are very small, then use of singular perturbations (Sauer \& Pai 1991) makes the $E_{d i}^{\prime}$ dynamics very fast so that (4) becomes an algebraic equation:

i.e.,

$$
0=-E_{d i}^{\prime}+\left(X_{q i}-X_{q i}^{\prime}\right) I_{q i}
$$

$$
E_{d i}^{\prime}=\left(X_{q i}-X_{q i}^{\prime}\right) I_{q i} \text {. }
$$

The differential and algebraic equations of the two-axis model will be modified by substituting (14) in (2), (8) and (9). Moreover, for a simple and fast exciter, the transfer function between $\left(V_{\text {ref.i } i}-V_{i}\right)$ and $E_{f d i}$ is represented by a single time constant, so that the exciter model becomes

$$
T_{A t}\left(\mathrm{~d} E_{f d i} / \mathrm{d} t\right)=-E_{f d i}+K_{A i}\left(V_{\text {res, } i}-V_{i}\right) .
$$

The overall flux decay model is represented by the following set of equations. 
2.2a Differential equations:

$$
\begin{aligned}
\frac{\mathrm{d} \delta_{i}}{\mathrm{~d} t} & =\omega_{i}-\omega_{s}, \\
\frac{\mathrm{d} \omega_{i}}{\mathrm{~d} t} & =\frac{T_{M i}}{M_{i}}-\frac{E_{q i}^{\prime} I_{q i}}{M_{i}}-\frac{\left(X_{q i}-X_{d i}^{\prime}\right)}{M_{i}} I_{d i} I_{q i} \frac{D_{i}\left(\omega_{i}-\omega_{s}\right)}{M_{i}}, \\
\frac{\mathrm{d} E_{q i}^{\prime}}{\mathrm{d} t} & =-\frac{E_{q i}^{\prime}}{T_{d o i}^{\prime}}-\frac{\left(X_{d i}-X_{d i}^{\prime}\right)}{T_{d o i}^{\prime}} I_{d i}+\frac{E_{f d i}}{T_{d o i}^{\prime}} \\
\frac{\mathrm{d} E_{f d i}}{\mathrm{~d} t} & =-\frac{E_{f d i}}{T_{A i}}+\frac{K_{A i}}{T_{A i}}\left(V_{r e f, i}-V_{i}\right), \quad \text { for } i=1, \ldots, m .
\end{aligned}
$$

2.2b Stator algebraic equations: The stator algebraic cquations are

$$
\begin{aligned}
& V_{i} \sin \left(\delta_{i}-\theta_{i}\right)+R_{s i} I_{d i}-X_{q i} I_{q i}=0, \\
& E_{q i}^{\prime}-V_{i} \cos \left(\delta_{i}-\theta_{i}\right)-R_{s i} I_{q i}-X_{d i}^{\prime} I_{d i}=0, \text { for } i=1, \ldots, m .
\end{aligned}
$$

The equivalent circuit for these equations is shown in figure 3.

2.2c Network equations: The network equations are the same as (10)-(13).

\subsection{Model C (two-axis model with fast exciter)}

In this model we have (1)-(4) as the differential equations for the machine and (8)-(13) as the algebraic equations. The exciter is represented by (15).

\subsection{Model D (flux decay with IEEE-type I exciter)}

The differential equations are (16)-(18) and (5)-(7). The algebraic equations are (20) (21) and (10)-(13).

\section{Linearization of model A}

Linearizing the differential equations (1) to (7) we have

$$
\begin{aligned}
\frac{\mathrm{d} \Delta \delta_{i}}{\mathrm{~d} t}= & \Delta \omega_{i} \\
\frac{\mathrm{d} \Delta \omega_{i}}{\mathrm{~d} t}= & \frac{1}{M_{i}} \Delta T_{M i}-\frac{E_{q i o}^{\prime}}{M_{i}} \Delta I_{q i}+\frac{X_{d i}^{\prime} I_{d i o}}{M_{i}} \Delta I_{q i}+\frac{X_{d i}^{\prime} I_{q i o}}{M_{i}} \Delta I_{d i}-\frac{I_{q i o}}{M_{i}} \Delta E_{q i}^{\prime} \\
& -\frac{E_{d i o}^{\prime}}{M_{i}} \Delta I_{d i}-\frac{I_{d i o}}{M_{i}} \Delta E_{d i}^{\prime}-\frac{X_{q i}^{\prime} I_{d i o}}{M_{i}} \Delta I_{q i}-\frac{X_{q i}^{\prime} I_{q i o}}{M_{i}} \Delta I_{d i}-\frac{D_{i}}{M_{i}} \Delta \omega_{i} \\
\frac{\mathrm{d} \Delta E_{q i}^{\prime}}{\mathrm{d} t}= & -\frac{\Delta E_{q i}^{\prime}}{T_{d o i}^{\prime}}-\frac{\left(X_{d i}-X_{d i}^{\prime}\right) \Delta I_{d i}}{T_{d o i}^{\prime}}+\frac{\Delta E_{f d i}}{T_{d o i}^{\prime}} \\
\frac{\mathrm{d} \Delta E_{d i}^{\prime}}{\mathrm{d} t}= & -\frac{\Delta E_{d i}^{\prime}}{T_{d o i}^{\prime}}+\frac{\left(X_{q i}-X_{q i}^{\prime}\right)}{T_{q o i}^{\prime}} \Delta I_{q i},
\end{aligned}
$$




$$
\begin{aligned}
& \frac{\mathrm{d} \Delta E_{f i}{ }_{d i}}{\mathrm{~d} t}=f_{i}\left(E_{f d i o}\right) \Delta E_{f d i}+\frac{\Delta V_{R i}}{T_{E i}}, \\
& \frac{\mathrm{d} \Delta V_{R i}}{\mathrm{~d} t}=-\frac{\Delta V_{R i}}{T_{A i}}+\frac{K_{A i}}{T_{A i}} \Delta R_{F i}-\frac{K_{A i} K_{F i}}{T_{A i} T_{F i}} \Delta E_{f d i}-\frac{K_{A i}}{T_{A i}} \Delta V_{i}+\frac{K_{A i}}{T_{A i}} \Delta V_{r e f, i}, \\
& \frac{\mathrm{d} \Delta R_{F i}}{\mathrm{~d} t}=-\frac{\Delta R_{F i}}{T_{F i}}+\frac{K_{F i}}{\left(T_{F i}\right)^{2}} \Delta E_{f \mathrm{di}}, \text { for } i=1, \ldots, m,
\end{aligned}
$$

where $f_{i}\left(E_{f d i o}\right)=-\left[K_{E i}+E_{f d i o} \Delta S_{E}\left(E_{\text {fdio }}\right)+S_{E}\left(E_{f d i o}\right)\right] / T_{E i}$. Writing equations (22) through (27) in matrix notation we obtain,

$\left[\begin{array}{l}\Delta \dot{\delta}_{i} \\ \Delta \dot{\omega}_{i} \\ \Delta \dot{E}_{q i}^{\prime} \\ \Delta \dot{E}_{d i}^{\prime} \\ \Delta \dot{E}_{f d i} \\ \Delta \dot{V}_{R i} \\ \Delta \dot{R}_{F i}\end{array}\right]=\left[\begin{array}{ccccccc}0 & 1 & 0 & 0 & 0 & 0 & 0 \\ 0 & -\frac{D_{i}}{M_{i}} & -\frac{I_{q i o}}{M_{i}} & -\frac{I_{d i o}}{M_{i}} & 0 & 0 & 0 \\ 0 & 0 & -\frac{1}{T_{d o i}^{\prime}} & 0 & \frac{1}{T_{d o i}^{\prime}} & 0 & 0 \\ 0 & 0 & 0 & -\frac{1}{T_{q o i}^{\prime}} & 0 & 0 & 0 \\ 0 & 0 & 0 & 0 & -\frac{K_{A i} K_{F i}}{T_{A i} T_{F i}} & -\frac{1}{T_{A i}} & \frac{K}{T_{A i}} \\ 0 & 0 & 0 & 0 & \frac{K_{F i}}{\left(T_{F i}\right)^{2}} & 0 & -\frac{1}{T_{F i}} \\ 0 & 0 & 0 & 0 & 0 \\ \Delta \omega_{i} \\ \Delta E_{q i}^{\prime} \\ \Delta E_{d i}^{\prime} \\ \Delta E_{f d i} \\ \Delta V_{R i} \\ \Delta R_{F i}\end{array}\right]$

$$
+\left[\begin{array}{cc}
0 & 0 \\
\frac{I_{q i o}\left(X_{d i}^{\prime}-X_{q i}^{\prime}\right)-E_{d i o}^{\prime}}{M_{i}} & \frac{I_{d i o}\left(X_{d i}^{\prime}-X_{q i}^{\prime}\right)-E_{q i o}^{\prime}}{M_{i}} \\
-\frac{\left(X_{d i}-X_{d i}^{\prime}\right)}{T_{d o i}^{\prime}} & 0 \\
0 & \frac{\left(X_{q i}-X_{q i}^{\prime}\right)}{T_{q o i}^{\prime}} \\
0 & 0 \\
0 & 0 \\
0 & 0
\end{array}\right]\left[\begin{array}{l}
\Delta I_{d i} \\
\Delta I_{q i}
\end{array}\right]
$$




$$
+\left[\begin{array}{cc}
0 & 0 \\
0 & 0 \\
0 & 0 \\
0 & 0 \\
0 & 0 \\
0 & -\frac{K}{T_{A i}} \\
0 & 0
\end{array}\right]\left[\begin{array}{c}
\Delta \theta_{i} \\
\Delta V_{i}
\end{array}\right]+\left[\begin{array}{cc}
0 & 0 \\
\frac{1}{M_{i}} & 0 \\
0 & 0 \\
0 & 0 \\
0 & 0 \\
0 & \frac{K A i}{T_{A i}} \\
0 & 0
\end{array}\right]\left[\begin{array}{c}
\Delta T_{M i} \\
\Delta V_{r e s . i}
\end{array}\right], i=1,2, \ldots, m
$$

Denoting $\left[\begin{array}{l}\Delta I_{d i} \\ \Delta I_{q i}\end{array}\right]=\Delta I_{g i},\left[\begin{array}{l}\Delta \theta_{i} \\ \Delta V_{i}\end{array}\right]=\Delta V_{g i}, \quad$ and $\left[\begin{array}{l}\Delta T_{M i} \\ \Delta V_{r e f, i}\end{array}\right]=\Delta u_{i}$,

we obtain,

$$
\Delta \dot{X}_{i}=A_{1 i} \Delta X_{i}+A_{2 i} \Delta I_{g i}+A_{3 i} \Delta V_{g i}+E_{i} \Delta u_{i}, \quad \text { for } i=1, \ldots, m
$$

For the $m$ machine system, (29) can be expressed as

$$
\Delta \dot{X}=A_{1} \Delta X+A_{2} \Delta I_{g}+A_{3} \Delta V_{g}+E \Delta U
$$

where $A_{1}, A_{2}, A_{3}$, and $E$ are block diagonal matrices. We now linearize the stator algebraic equations (8) and (9) to get

$$
\begin{aligned}
\Delta E_{d i}^{\prime}- & \sin \left(\delta_{i o}-\theta_{i o}\right) \Delta V_{i}-V_{i o} \cos \left(\delta_{i o}-\theta_{i o}\right) \Delta \delta_{i}+V_{i o} \cos \left(\delta_{i o}-\theta_{i o}\right) \Delta \theta_{i} \\
& -R_{s i} \Delta I_{d i}+X_{q i}^{\prime} \Delta I_{q i}=0, \\
\Delta E_{q i}^{\prime}- & \cos \left(\delta_{i o}-\theta_{i o}\right) \Delta V_{i}+V_{i o} \sin \left(\delta_{i o}-\theta_{i o}\right) \Delta \delta_{i}-V_{i o} \sin \left(\delta_{i o}-\theta_{i o}\right) \Delta \theta_{i} \\
& -R_{s i} \Delta I_{q i}-X_{d i}^{\prime} \Delta I_{d i}=0, \quad i=1,2, \ldots, m .
\end{aligned}
$$

Writing (31) and (32) in the matrix form, we have

$$
\begin{aligned}
& {\left[\begin{array}{ccccccc}
-V_{i o} \cos \left(\delta_{i o}-\theta_{i o}\right) & 0 & 0 & 1 & 0 & 0 & 0 \\
V_{i o} \sin \left(\delta_{i o}-\theta_{i o}\right) & 0 & 1 & 0 & 0 & 0 & 0
\end{array}\right]} \\
& {\left[\begin{array}{l}
\Delta \delta_{i} \\
\Delta \omega_{i} \\
\Delta E_{q i}^{\prime} \\
\Delta E_{d i}^{\prime} \\
\Delta E_{f d i} \\
\Delta V_{R i} \\
\Delta R_{F i}
\end{array}\right]+\left[\begin{array}{cr}
-R_{s i} & X_{q i}^{\prime} \\
-X_{d i}^{\prime} & -R_{s i}
\end{array}\right]\left[\begin{array}{l}
\Delta I_{d i} \\
\Delta I_{q i}
\end{array}\right]+\left[\begin{array}{ll}
V_{i o} \cos \left(\delta_{i o}-\theta_{i o}\right) & -\sin \left(\delta_{i o}-\theta_{i o}\right) \\
-V_{i o} \sin \left(\delta_{i o}-\theta_{i o}\right)-\cos \left(\delta_{i o}-\theta_{i o}\right)
\end{array}\right]} \\
& {\left[\begin{array}{l}
\Delta \theta_{i} \\
\Delta V_{i}
\end{array}\right]=0, \quad i=1,2, \ldots, m \text {. }}
\end{aligned}
$$


Rewriting (33) we get

$$
0=B_{1 i} \Delta X_{i}+B_{2 i} \Delta I_{g i}+B_{3 i} \Delta V_{g i}, \quad i=1, \ldots, m .
$$

In compact notation, (34) can be written as

$$
0=B_{1} \Delta X+B_{2} \Delta I_{g}+B_{3} \Delta V_{g}
$$

where $B_{1}, B_{2}$, and $B_{3}$ are block diagonal matrices. Linearizing the network equations (10) and (11), which pertain to generators, we obtain

$$
\begin{aligned}
& V_{i o} \sin \left(\delta_{i o}-\theta_{i o}\right) \Delta I_{d i}+I_{d i o} \sin \left(\delta_{i o}-\theta_{i o}\right) \Delta V_{i}+I_{d i o} V_{i o} \cos \left(\delta_{i o}-\theta_{i o}\right) \Delta \delta_{i} \\
& -I_{d i 0} V_{i 0} \cos \left(\delta_{i 0}-\theta_{i 0}\right) \Delta \theta_{i}+V_{i 0} \cos \left(\delta_{i 0}-\theta_{i 0}\right) \Delta I_{q i}+I_{q i 0} \cos \left(\delta_{i 0}-\theta_{i 0}\right) \Delta V_{i} \\
& -\left[\sum_{k=1}^{n} V_{k o} Y_{i k} \cos \left(\theta_{i o}-\theta_{k o}-\alpha_{i k}\right)\right] \Delta V_{i} \\
& -V_{i o} \sum_{k=1}^{n}\left[Y_{i k} \cos \left(\theta_{i o}-\theta_{k o}-\alpha_{i k}\right)\right] \Delta V_{k} \\
& +\left[V_{i o} \sum_{\substack{k=1 \\
\neq i}} V_{k o} Y_{i k} \sin \left(\theta_{i o}-\theta_{k o}-\alpha_{i k}\right)\right] \Delta \theta_{i} \\
& -V_{i o} \sum_{\substack{k=1 \\
\neq i}}^{n}\left[V_{k o} Y_{i k} \sin \left(\theta_{i o}-\theta_{k o}-\alpha_{i k}\right)\right] \Delta \theta_{k}+\Delta P_{L i}\left(V_{i}\right)=0 \\
& V_{i o} \cos \left(\delta_{i 0}-\theta_{i 0}\right) \Delta I_{d i}+I_{d i o} \cos \left(\delta_{i 0}-\theta_{i 0}\right) \Delta V_{i}-I_{d i o} V_{i o} \sin \left(\delta_{i 0}-\theta_{i 0}\right) \Delta \delta_{i} \\
& +I_{d i o} V_{i o} \sin \left(\delta_{i o}-\theta_{i o}\right) \Delta \theta_{i}-V_{i o} \sin \left(\delta_{i o}-\theta_{i o}\right) \Delta I_{q i}-I_{q i o} \sin \left(\delta_{i o}-\theta_{i o}\right) \Delta V_{i} \\
& -I_{q i o} V_{i o} \cos \left(\delta_{i o}-\theta_{i o}\right) \Delta \delta_{i}+I_{q i o} V_{i o} \cos \left(\delta_{i o}-\theta_{i o}\right) \Delta \theta_{i} \\
& -\left[\sum_{k=1}^{n} V_{k o} Y_{i k} \sin \left(\theta_{i o}-\theta_{k o}-\alpha_{i k}\right)\right] \Delta V_{i} \\
& -V_{i o} \sum_{k=1}^{n}\left[Y_{i k} \sin \left(\theta_{i o}-\theta_{k o}-\alpha_{i k}\right)\right] \Delta V_{k}-\left[V_{i o} \sum_{\substack{k=1 \\
\neq i}}^{n} V_{k o} Y_{i k} \cos \left(\theta_{i o}-\theta_{k o}-\alpha_{i k}\right)\right] \Delta \theta_{i} \\
& +V_{i o} \sum_{\substack{k=1 \\
\neq i}}^{n}\left[V_{k o} Y_{i k} \cos \left(\theta_{i o}-\theta_{k o}-\alpha_{i k}\right)\right] \Delta \theta_{k}+\Delta Q_{L i}\left(V_{i}\right)=0, \quad i=1,2, \ldots, m .
\end{aligned}
$$

Rewriting (36) and (37), we obtain

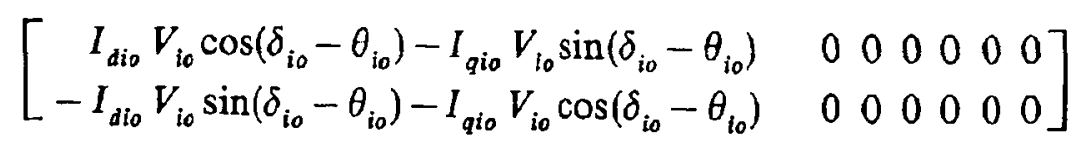$$
\left[\begin{array}{l}
\Delta \delta_{i} \\
\Delta \omega_{i} \\
\Delta E_{q i}^{\prime} \\
\Delta E_{d i}^{\prime} \\
\Delta E_{f d i} \\
\Delta V_{R i} \\
\Delta R_{F i}
\end{array}\right]+\left[\begin{array}{rr}
V_{i o} \sin \left(\delta_{i o}-\theta_{i 0}\right) & V_{i o} \cos \left(\delta_{i o}-\theta_{i o}\right) \\
V_{i o} \cos \left(\delta_{i o}-\theta_{i o}\right) & -V_{i o} \sin \left(\delta_{i 0}-\theta_{i o}\right)
\end{array}\right]\left[\begin{array}{l}
\Delta I_{d i} \\
\Delta I_{q i}
\end{array}\right]
$$ 
$+\left[\begin{array}{cc}I_{q i o} V_{i o} \sin \left(\delta_{i o}-\theta_{i o}\right) & I_{d i o} \sin \left(\delta_{i o}-\theta_{i o}\right) \\ -I_{d i o} V_{i o} \cos \left(\delta_{i o}-\theta_{i o}\right) & +I_{q i o} \cos \left(\delta_{i o}-\theta_{i o}\right) \\ +V_{i o} \sum_{\substack{k=1 \\ \neq i}}^{n} V_{k o} Y_{i k} \sin \left(\theta_{i o}-\theta_{k o}-\alpha_{i k}\right) & -\sum_{\substack{k=1 \\ \neq i}}^{n} V_{k o} Y_{i k} \cos \left(\theta_{i o}-\theta_{k o}-\alpha_{i k}\right) \\ I_{d i o} V_{i o} \sin \left(\delta_{i o}-\theta_{i o}\right)+I_{q i o} V_{i o} \cos \left(\delta_{i o}-\theta_{i o}\right) & -V_{i o} Y_{i i} \cos \alpha_{i i} \\ -V_{i o} \sum_{\substack{k=1 \\ \neq i}}^{n} V_{k o} Y_{i k} \cos \left(\theta_{i o}-\theta_{k o}-\alpha_{i k}\right) & -\sum_{\substack{k=1 \\ \neq i}}^{n} V_{k o} Y_{i k} \sin \left(\theta_{i o}-\theta_{k o}-\alpha_{i k}\right)\end{array}\right]$

$\left[\begin{array}{c}\Delta \theta_{i} \\ \Delta V_{i}\end{array}\right]+\sum_{\substack{k=1 \\ \neq i}}^{m}\left[\begin{array}{cc}-V_{i o} V_{k o} Y_{i k} \sin \left(\theta_{i o}-\theta_{k o}-\alpha_{i k}\right) & -V_{i o} Y_{i k} \cos \left(\theta_{i o}-\theta_{k o}-\alpha_{i k}\right) \\ V_{i o} V_{k o} Y_{i k} \cos \left(\theta_{i o}-\theta_{k o}-\alpha_{i k}\right) & -V_{i o} Y_{i k} \sin \left(\theta_{i o}-\theta_{k o}-\alpha_{i k}\right)\end{array}\right]\left[\begin{array}{c}\Delta \theta_{k} \\ \Delta V_{k}\end{array}\right]$ $+\left[\begin{array}{c}\Delta P_{L i}\left(V_{i}\right) \\ \Delta Q_{L i}\left(V_{i}\right)\end{array}\right]+\sum_{j=m+1}^{n}\left[\begin{array}{c}-V_{i o} V_{j o} Y_{i j} \sin \left(\theta_{i o}-\theta_{j o}-\alpha_{i j}\right)-V_{i o} Y_{i j} \cos \left(\theta_{i o}-\theta_{j o}-\alpha_{i j}\right) \\ V_{i o} V_{j o} Y_{i j} \cos \left(\theta_{i o}-\theta_{j o}-\alpha_{i j}\right)-V_{i o} Y_{i j} \sin \left(\theta_{i o}-\theta_{j o}-\alpha_{i j}\right)\end{array}\right]$ $\left[\begin{array}{l}\Delta \theta_{j} \\ \Delta V_{j}\end{array}\right]=0$

Rearranging in matrix notation

$$
\begin{aligned}
0= & C_{1 i} \Delta X_{i}+C_{2 i} \Delta I_{g i}+\left[C_{3 i 1} C_{3 i 2} \cdots C_{3 i m}\right]\left[\begin{array}{c}
\Delta V_{g 1} \\
\Delta V_{g 2} \\
\vdots \\
\Delta V_{g m}
\end{array}\right] \\
& +\left[C_{4 i m} C_{4 i, m+1} \cdots C_{4 i n}\right]\left[\begin{array}{c}
\Delta V_{l, m+1} \\
\Delta V_{l, m+2} \\
\vdots \\
\Delta V_{l, n}
\end{array}\right]+\left[\begin{array}{c}
\Delta P_{L i}\left(V_{i}\right) \\
\Delta Q_{L i}\left(V_{i}\right)
\end{array}\right], \text { for } i=1, \ldots, m .
\end{aligned}
$$

For $m$ machines, (39) can be written in matrix notation as

$$
\begin{aligned}
& 0=\left[\begin{array}{cccc}
C_{11} & & & \\
& C_{12} & & \\
& & \ddots & \\
& & & C_{1 m}
\end{array}\right]\left[\begin{array}{c}
\Delta X_{1} \\
\Delta X_{2} \\
\vdots \\
\Delta X_{m}
\end{array}\right]+\left[\begin{array}{llll}
C_{21} & & & \\
& C_{22} & \\
& & \ddots & \\
& & & C_{2 m}
\end{array}\right]\left[\begin{array}{c}
\Delta I_{g 1} \\
\Delta I_{g 2} \\
\vdots \\
\Delta I_{g m}
\end{array}\right] \\
& +\left[\begin{array}{cccc}
C_{3,11} & C_{3,12} & \cdots & C_{3,1 m} \\
\vdots & \vdots & \vdots & \vdots \\
C_{3, m 1} & C_{3, m 2} & \cdots & C_{3, m m}
\end{array}\right]\left[\begin{array}{c}
\Delta V_{g 1} \\
\Delta V_{g 2} \\
\vdots \\
\Delta V_{g m m}
\end{array}\right]+\left[\begin{array}{cccc}
C_{4,1 m+1} & C_{4,1 m+2} & \cdots & C_{4,1 n} \\
C_{4,2 m+1} & C_{4,2 m+2} & \cdots & C_{4,2 n} \\
\vdots & \vdots & \vdots & \vdots \\
C_{4, m m+1} & C_{4, m m+2} & \cdots & C_{4, m n}
\end{array}\right]
\end{aligned}
$$




$$
\left[\begin{array}{c}
\Delta V_{l m+1} \\
\Delta V_{l m+2} \\
\vdots \\
\Delta V_{l n}
\end{array}\right]+\left[\begin{array}{c}
\Delta P_{L 1}\left(V_{i}\right) \\
\Delta Q_{L 1}\left(V_{i}\right) \\
\vdots \\
\Delta P_{L m}\left(V_{m}\right) \\
\Delta Q_{L m}\left(V_{m}\right)
\end{array}\right]
$$

Rewriting (40) as

$$
0=C_{1} \Delta \dot{X}+C_{2} \Delta I_{g}+C_{3} \Delta V_{g}+C_{4} \Delta V_{l}+\Delta S_{L_{g}}(V) .
$$

Note that $C_{1}, C_{2}$ are block diagonal whereas $C_{3}, C_{4}$ are full matrices. Linearizing network equations (12) and (13) for load buses

$$
\begin{aligned}
0= & \Delta P_{L i}\left(V_{i}\right)-\left[\sum_{k=1}^{n} V_{k o} Y_{i k} \cos \left(\theta_{i o}-\theta_{k o}-\alpha_{i k}\right)\right] \Delta V_{i} \\
& +\left[\sum_{\substack{k=1 \\
\neq i}}^{n} V_{i o} V_{k o} Y_{i k} \sin \left(\theta_{i o}-\theta_{k o}-\alpha_{i k}\right)\right] \Delta \theta_{i} \\
& -V_{i o} \sum_{k=1}^{n}\left[Y_{i k} \cos \left(\theta_{i o}-\theta_{k o}-\alpha_{i k}\right)\right] \Delta V_{k} \\
& -V_{i o} \sum_{\substack{k=1 \\
\neq i}}^{n}\left[V_{k o} Y_{i k} \sin \left(\theta_{i o}-\theta_{k o}-\alpha_{i k}\right)\right] \Delta \theta_{k}, \\
0= & \Delta Q_{L i}\left(V_{i}\right)-\left[\sum_{k=1}^{n} V_{k o} Y_{i k} \sin \left(\theta_{i o}-\theta_{k o}-\alpha_{i k}\right)\right] \Delta V_{i} \\
& -\left[\sum_{\substack{k=1 \\
\neq i}}^{n} V_{i o} V_{k o} Y_{i k} \cos \left(\theta_{i o}-\theta_{k o}-\alpha_{i k}\right)\right] \Delta \theta_{i} \\
& -V_{i o} \sum_{\substack{k=1 \\
j}}^{n}\left[Y_{i k} \sin \left(\theta_{i o}-\theta_{k o}-\alpha_{i k}\right)\right] \Delta V_{k} \\
& +V_{i o} \sum_{\substack{k=1 \\
\neq i}}^{n}\left[V_{k o} Y_{i k} \sin \left(\theta_{i o}-\theta_{k o}-\alpha_{i k}\right)\right] \Delta \theta_{k}, \quad i=m+1, \ldots, n .
\end{aligned}
$$

Rewriting (42) and (43) we obtain

$$
\begin{aligned}
& 0=\left[\begin{array}{l}
\Delta P_{L i}\left(V_{j}\right) \\
\Delta Q_{L i}\left(V_{i}\right.
\end{array}\right] \\
&+ \sum_{j=1}^{m}\left[\begin{array}{c}
-V_{i o} V_{j o} Y_{i j} \sin \left(\theta_{i o}-\theta_{j o}-\alpha_{i j}\right)-V_{i o} Y_{i j} \cos \left(\theta_{i o}-\theta_{j o}-\alpha_{i j}\right) \\
V_{i o} V_{j o} Y_{i j} \cos \left(\theta_{i o}-\theta_{j o}-\alpha_{i j}\right)-V_{i o} Y_{i j} \sin \left(\theta_{i o}-\theta_{j o}-\alpha_{i j}\right)
\end{array}\right]\left[\begin{array}{l}
\Delta \theta_{j} \\
\Delta V_{j}
\end{array}\right] \\
&+\sum_{\substack{k=m+1 \\
\neq i}}^{n}\left[\begin{array}{c}
-V_{i o} V_{k o} Y_{i k} \sin \left(\theta_{i o}-\theta_{k o}-\alpha_{i k}\right)-V_{i o} Y_{i k} \cos \left(\theta_{i o}-\theta_{k o}-\alpha_{i k}\right) \\
V_{i o} V_{k o} Y_{i k} \cos \left(\theta_{i o}-\theta_{k o}-\alpha_{i k}\right)-V_{i o} Y_{i k} \sin \left(\theta_{i o}-\theta_{k o}-\alpha_{i k}\right)
\end{array}\right]\left[\begin{array}{l}
\Delta \theta_{k} \\
\Delta V_{k}
\end{array}\right]
\end{aligned}
$$




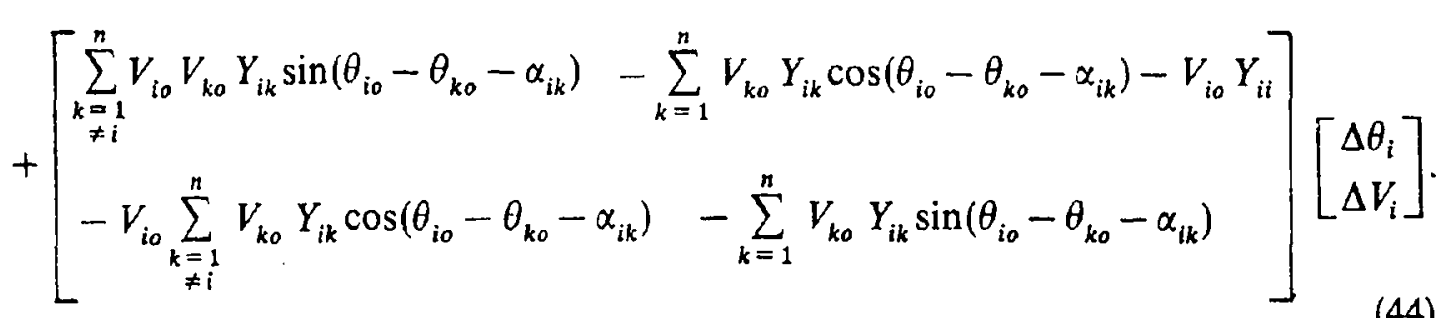

Writing the above equation for all load buses in matrix notation, we obtain

$$
\begin{aligned}
0= & {\left[\begin{array}{c}
\Delta P_{L m+1}\left(V_{m+1}\right) \\
\Delta Q_{L m+1}\left(V_{m+1}\right) \\
\vdots \\
\Delta P_{L n}\left(V_{n}\right) \\
\Delta Q_{L n}\left(V_{n}\right)
\end{array}\right]+\left[\begin{array}{cccc}
D_{1, m+1,1} & D_{1, m+1,2} & \cdots & D_{1, m+1, m} \\
D_{1, m+2,1} & D_{1, m+2,2} & \cdots & D_{1, m+2, m} \\
\vdots & \vdots & \vdots & \vdots \\
D_{1, n, 1} & D_{1, n, 2} & \cdots & D_{1, n, m}
\end{array}\right]\left[\begin{array}{c}
\Delta V_{\theta 1} \\
\Delta V_{g 2} \\
\vdots \\
\Delta V_{g m}
\end{array}\right] } \\
& +\left[\begin{array}{cccc}
D_{2, m+1, m+1} & D_{2, m+1, m+2} & \cdots & D_{2, m+1, n} \\
D_{2, m+2, m+1} & D_{2, m+2, m+2} & \cdots & D_{2, m+2, n} \\
\vdots & \vdots & \vdots & \vdots \\
D_{2, n, m+1} & D_{2, n, m+2} & \cdots & D_{2, n, n}
\end{array}\right]\left[\begin{array}{c}
\Delta V_{l m+1} \\
\Delta V_{l m+2} \\
\vdots \\
\Delta V_{l n}
\end{array}\right] .
\end{aligned}
$$

Rewriting (45) in a compact notation we obtain

$$
0=\Delta S_{L l}(V)+D_{1} \Delta V_{g}+D_{2} \Delta V_{l} .
$$

Note that $D_{1}$ and $D_{2}$ are full matrices. Rewriting (30), (35), (41) and (46) together, we obtain

$$
\begin{aligned}
\Delta \dot{X} & =A_{1} \Delta X+A_{2} \Delta I_{g}+A_{3} \Delta V_{g}+E \Delta U, \\
0 & =B_{1} \Delta X+B_{2} \Delta I_{g}+B_{3} \Delta V_{g}, \\
0 & =C_{1} \Delta X+C_{2} \Delta I_{g}+C_{3} \Delta V_{g}+C_{4} \Delta V_{l}+\Delta S_{L g}(V), \\
0 & =D_{1} \Delta V_{\theta}+D_{2} \Delta V_{l}+\Delta S_{L l}(V) .
\end{aligned}
$$

This is the general comprehensive model of the differential-algebraic type to study both steady state and voltage stabilities with any type of nonlinear voltage dependent loads. The network structure is preserved and so are the stator algebraic equations for each machine. Equations (47)-(50) are equivalent to the model in Sauer \& Pai (1990) except that a machine angle is not introduced as reference. This model is quite general and can easily be expanded to include frequency or $\dot{V}$ dependence at the load buses, PSS tap-changer dynamics or FACTS devices. This will only augment (47)-(50) by either algebraic or differential equations. In the above model, $\Delta I_{g}$ is not of interest in most cases. Hence, eliminating $\Delta I_{g}$ from the set of equations (47), (48) 
and (49) we get the following reduced set of equations,

$$
\begin{aligned}
\Delta \dot{X} & =\left(A_{1}-A_{2} B_{2}^{-1} B_{1}\right) \Delta X+\left(A_{3}-A_{2} B_{2}^{-1} B_{3}\right) \Delta V_{g}+E \Delta U, \\
0 & =K_{2} \Delta X+K_{1} \Delta V_{g}+C_{4} \Delta V_{1}+\Delta S_{L g}(V), \\
0 & =D_{1} \Delta V_{g}+D_{2} \Delta V_{l}+\Delta S_{L l}(V),
\end{aligned}
$$

where

and

$$
\left[C_{3}-C_{2} B_{2}^{-1} B_{3}\right] \triangleq K_{1}
$$

$$
\left[C_{1}-C_{2} B_{2}^{-1} B_{1}\right] \triangleq K_{2} \text {. }
$$

More compactly, (51)-(53) can be put in the form

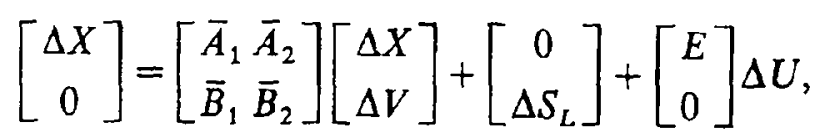

where $\Delta V=\left[\begin{array}{l}\Delta V_{g} \\ \Delta V_{l}\end{array}\right]$ and $\Delta S_{L}=\left[\begin{array}{l}\Delta S_{L g} \\ \Delta S_{L l}\end{array}\right]$. Reorder the variables in the vector $\Delta V=$ $\left[\begin{array}{c}\Delta V_{g} \\ \Delta V_{l}\end{array}\right]$ such that the new vector is $\left[\Delta z^{T} \mid \Delta v^{T}\right]=\left[\Delta \theta_{1}, \Delta V_{1}, \ldots, \Delta V_{m} \mid \Delta \theta_{2}, \Delta 0_{3}, \ldots, \Delta \theta_{n}\right.$, $\left.\Delta V_{m+1}, \ldots, \Delta V_{n}\right]$. In this reordering of algebraic variables $\Delta v$ represents those variables appearing in the standard load flow equations and $\Delta z$ the remaining ones in $\Delta V$. Also reorder the variables in $\left[\begin{array}{l}\Delta S_{L g} \\ \Delta S_{L l}\end{array}\right]$ to conform similarly so that $\Delta S_{L}^{T}=\left[\Delta P_{L 1}, \Delta Q_{L 1}, \ldots\right.$, $\left.\Delta Q_{L m} \mid \Delta P_{L 2}, \Delta P_{L 3}, \ldots, \Delta P_{L n}, \Delta Q_{L m+1}, \ldots, \Delta Q_{L n}\right]=\left[\Delta S_{1}^{T} \mid \Delta S_{2}^{T}\right]$. We carry out one more operation on the set of equations (51)-(53). In any rotational system, the reference for angles is arbitrary. The order of the dynamical system in (51) is $7 \mathrm{~m}$, and can be reduced to $(7 m-1)$ by introducing relative rotor angles (Sauer \& Pai 1990). Selecting $\delta_{1}$ as the reference, we have

$$
\begin{aligned}
& \delta_{i}^{\prime}=\delta_{i}-\delta_{1}, \quad i=2,3, \ldots, m, \\
& \delta_{1}^{\prime}=0, \\
& \dot{\delta}_{i}^{\prime}=\omega_{i}-\omega_{1}, \quad i=2,3, \ldots, m, \\
& \dot{\delta}_{1}^{\prime}=0, \\
& \theta_{i}^{\prime}=\theta_{i}-\delta_{1}, \quad i=1,2, \ldots, n .
\end{aligned}
$$

This implies that the differential equation corresponding to $\delta_{1}$ can be deleted from (55) and also the column corresponding to $\Delta \delta_{1}$ in $\bar{A}_{1}$ and $\bar{B}_{1}$. Moreover, the entries corresponding to $\dot{\delta}_{i}^{\prime}, i=2,3, \ldots, m$ will bring necessary changes in $\bar{A}_{1}$. We denote the reduced state vector as $\Delta x$. We thus have the new differential-algebraic (DAE) system as

$$
\left[\begin{array}{c}
\Delta \dot{x} \\
0 \\
0
\end{array}\right]=\left[\begin{array}{lll}
A_{1} & A_{2} & A_{3} \\
B_{1} & B_{2} & B_{3} \\
C_{1} & C_{2} & C_{3}
\end{array}\right]\left[\begin{array}{c}
\Delta x \\
\Delta z \\
\Delta v
\end{array}\right]+\left[\begin{array}{c}
0 \\
\Delta S_{1} \\
\Delta S_{2}
\end{array}\right]+\left[\begin{array}{l}
E \\
0 \\
0
\end{array}\right] \Delta U
$$

This model is slightly different from that in Sauer \& Pai (1990) in the sense that we 
allow for voltage dependency for the loads in the vectors $\Delta S_{1}$ and $\Delta S_{2}$. For the constant power case, both $\Delta S_{1}$ and $\Delta S_{2}$ are $\equiv 0$. Otherwise, $\Delta S_{1 i}=\Delta S_{1 i}\left(V_{i}\right)$ and $\Delta S_{2 i}=\Delta S_{2 i}\left(V_{i}\right)$. For a given voltage-dependent load, $\Delta S_{1 i}\left(V_{i}\right)$ and $\Delta S_{2 i}\left(V_{i}\right)$ can be computed. Only the appropriate diagonal elements of $B_{2}, C_{2}$ and $C_{3}$ will be modified and we obtain the system

$$
\left[\begin{array}{c}
\Delta \dot{x} \\
0 \\
0
\end{array}\right]=\left[\begin{array}{lll}
\tilde{A}_{1} & \tilde{A}_{2} & \tilde{A}_{3} \\
\tilde{B}_{1} & \tilde{B}_{2} & \tilde{B}_{3} \\
\tilde{C}_{1} & \tilde{C}_{2} & \tilde{C}_{3}
\end{array}\right]\left[\begin{array}{c}
\Delta x \\
\Delta z \\
\Delta v
\end{array}\right]+\left[\begin{array}{l}
E \\
0 \\
0
\end{array}\right] \Delta U .
$$

Now $\tilde{C}_{3}$ is the load flow Jacobian $J_{L F}$ and $\left[\begin{array}{cc}\tilde{B}_{2} & \bar{B}_{3} \\ \tilde{C}_{2} & \bar{C}_{3}\end{array}\right]=J_{A E}$ defined as the algebraic Jacobian. The system A matrix is obtained as

where

$$
\Delta \dot{x}=A_{s y s} \Delta x+E \Delta U,
$$

$$
A_{s y s}=\tilde{A}_{1}-\left[\tilde{A}_{2} \tilde{A}_{3}\right]\left[J_{A E}\right]^{-1}\left[\begin{array}{l}
\tilde{B}_{1} \\
\tilde{C}_{1}
\end{array}\right] \text {. }
$$

This is the model used in studying low frequency oscillation, steady state stability and voltage stability. In the next section we examine the effects of increased loading on the eigenvalues of $A_{s y s}$ and the determinants of $J_{A E}$ and $J_{L F}$ for (a) constant power case, (b) constant current case, and (c) constant impedance case for each of the models A, B, C, and D. Thus we do the parametric study regarding load and generating unit models on small signal stability.

The linearized equations for models $\mathrm{B}, \mathrm{C}$, and $\mathrm{D}$ can be derived along the same lines and hence are omitted.

\section{Identification of critical modes: Participation factor method}

Due to the large size of the power system, it is often necessary to construct reducedorder models for dynamic stability studies. The appropriate definition and determination as to which state variables significantly participate in the selected modes become very important. This requires tools for identifying state variables that are significant in producing the selected modes. It is natural to suggest that the significant state variables for an eigenvalue $\lambda_{i}$ are those that correspond to large entries in the eigenvector $v_{i}$. But the entries in the eigenvector are dependent on the dimensions of the state variables, which are, in general, incommensurable (for example, angles, velocities, and flux). Verghese et al (1982) have suggested a related but dimensionless measure of state variable participation (henceforth called participation factors). If $v_{i}$ and $w_{i}$ represent the right- and left-hand eigenvectors, respectively, for the eigenvalue $\lambda_{i}$ of the matrix $A$, then the participation factor (PF) measuring the participation of the $k$ th state variable $x_{k}$ in the ith mode is defined as

$$
p_{k i}=w_{k i} v_{k i} \text {. }
$$

This quantity is dimensionless and hence invariant under changes of scale of the variables. We can think of $v_{k i}$ as measuring the activity of $x_{k}$ in the ith mode, and 
$w_{k i}$ as weighting the contribution of this activity to the mode. The step-by-step procedure for the calculation of PF is as follows:

(1) For the given $A_{s y s}$, the eigenvalues $\lambda_{i}^{\prime}$ s and the right- and the left-hand eigenvectors $\left(\mathscr{R}_{1}\right.$ and $\mathscr{L}_{i}$, respectively) are calculated, for $i=1,2, \ldots,(7 m-1)$.

(2) For a given eigenvalue $\lambda_{i}$ the corresponding PF are calculated as follows:
(a) The normalization constant $\mathscr{N}_{i}$ is calculated by finding $\sum_{j=1}^{N} a b s\left(\mathscr{R}_{i j}\right)$ abs $\left(\mathscr{L}_{i j}\right)$ where $N=7 m-1$.
(b) Then $\operatorname{PF}_{i j}=a b s\left(\mathscr{R}_{i j}\right) a b s\left(\mathscr{L}_{i j}\right) / \mathcal{N}_{i}$.

\section{Studies of parametric effects}

\subsection{Effect of loading}

The effect of loading has been investigated on the 3-machine, 9-bus system (figure 6) whose data can be found in Anderson \& Fouad (1977). The real and/or reactive loads at a particular bus/buses were increased continuously. At each step the initial conditions of the state variables were computed, after running the load flow (Sauer \& Pai 1990), and linearization of the equations was done. Ideally, the increase of load should be picked up by the generators through the economic load dispatch scheme. To simplify matters we allocate the increase in generation (real power) to the machines in proportion to the inertias. In the case of increase in reactive power, the increase is picked up by the PV buses. The $A_{\text {sys }}$ matrix was formed using (55) and its eigenvalues were checked for stability. Also det $J_{L F}$ and det $J_{A E}$ were computed. The step-by-step algorithm is as follows:

(a) increase the load at bus/buses for a particular generating unit model;

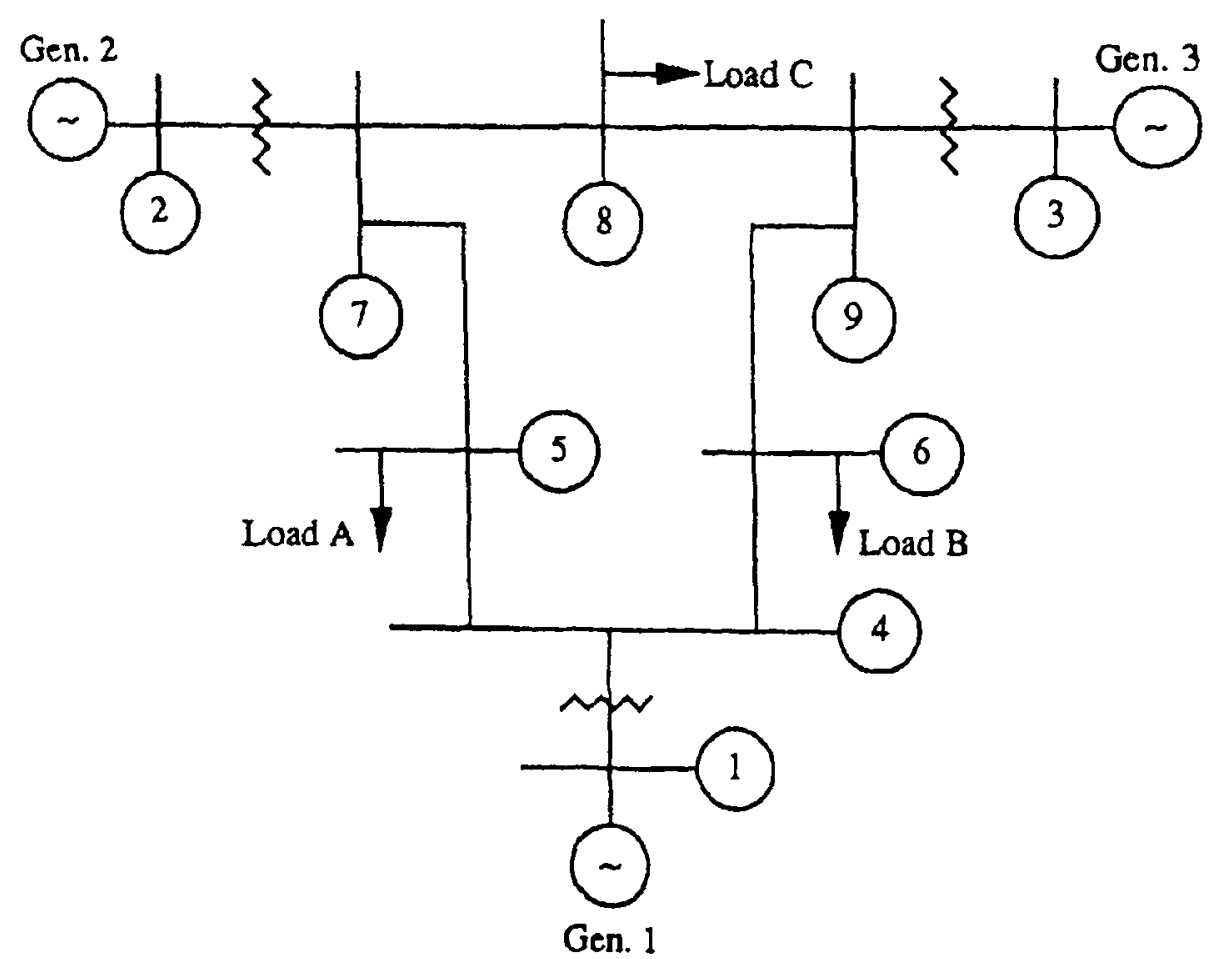

Figure 6. 3-Machine 9-bus system. 
(b) if the real load is increased then distribute the load amongst various generators in proportion to their inertias;

(c) run the load flow;

(d) stop, if load flow fails to converge;

(e) compute initial conditions of the state variables;

(f) linearise the differential equations and compute the various matrices;

(g) compute $\operatorname{det} J_{L F}$, det $J_{A E}$, and the eigenvalues of $A_{s y s}$;

(h) if $A_{\text {sys }}$ is stable then go to step (a);

(i) identify the states associated with the unstable eigenvalue (s) of $A_{s y s}$ using the participation factor method and go to step (a).

For the 3-machine case all the four generating units are studied. The results are summarized in tables (1)-(4) for the models A-D. For the static exciter, a gain of $K_{A}=50$ was assumed.

\subsection{Effect of $K_{A}$}

It was found that for model $\mathrm{A}$, the increase in $K_{A}$ alone did not lead to any instability. The stabilizing feedback in IEEE-type 1 exciter was removed and then an increase in $K_{A}$ led to instability for this model as well. For model $\mathrm{B}$ a sufficient increase in $K_{A}$ led to instability even for a nominal load.

\subsection{Effect of type of load}

The effect of different types of voltage-dependent loads has been studied in the following manner. From (55) we can obtain the following model by elementary matrix manipulation. Also the state vector is the reduced one, namely $x$, whose dimension is $(7 m-1)$.

$$
\begin{aligned}
& \Delta \dot{x}=A \Delta x+B \Delta S_{L}+E \Delta U, \\
& \Delta V=C \Delta x+D \Delta S_{L} .
\end{aligned}
$$

In our studies, we consider only nonlinear voltage-dependent loads. The load at any

\begin{tabular}{|c|c|c|c|c|}
\hline $\begin{array}{l}\text { Load at } \\
\text { bus } 5\end{array}$ & Sign $\left(\operatorname{det} J_{L F}\right)$ & $\operatorname{Sign}\left(\operatorname{det} J_{A E}\right)$ & Critical eigenvalue(s) & Associated states \\
\hline $4 \cdot 3$ & + & + & $-0.1433 \pm j 2.0188$ & $E_{a 1}^{\prime} \& R_{11}$ \\
\hline $4 \cdot 4$ & + & + & $0.0057 \pm j 2.2434$ & $E_{q 1}^{q_{1}^{1}} \& R_{f 1}^{J 1}$ \\
\hline $4 \cdot 5$ & + & + & $0.3400 \pm j 2.5538$ & $E_{Q 1}^{g 1} \& R$ \\
\hline $4 \cdot 6$ & + & + & $1 \cdot 1350 \pm j 2 \cdot 8016$ & $E_{q 1}^{g 1} \& R_{f 1}^{J 1}$ \\
\hline $4 \cdot 7$ & + & + & $2 \cdot 5961 \pm j 2 \cdot 2768$ & $E_{q 1}^{q+} \& R_{f 1}$ \\
\hline 4.8 & + & + & $9 \cdot 2464,1 \cdot 8176$ & $\delta_{2} \& \omega_{2}, E_{21}^{\prime} \& R_{f 1}$ \\
\hline $4 \cdot 9$ & + & - & 1.0542 & $E_{q 1}^{\prime} \& R_{f 1}^{21}$ \\
\hline $5 \cdot 0$ & + & - & $0-6298$ & $E_{q 1}^{\prime A} \& R_{f 1}$ \\
\hline $5 \cdot 1$ & + & - & 0.2463 & $E_{t 1}^{\prime 1} \& R_{f 1}^{J 1}$ \\
\hline $5 \cdot 15$ & + & - & -0.6832 & $E_{q 1}^{q^{1}} \& R_{f 1}$ \\
\hline 5.2 & \multicolumn{4}{|c|}{ Load flow does not converge } \\
\hline
\end{tabular}

Table 1. Modal behaviour of model $\mathrm{A}$ for different loads. 
Table 2. Modal behaviour of model B for different loads.

\begin{tabular}{|c|c|c|c|c|}
\hline $\begin{array}{l}\text { Load at } \\
\text { bus } 5\end{array}$ & $\begin{array}{c}\text { Sign } \\
\left(\operatorname{det} J_{L F}\right)\end{array}$ & $\begin{array}{c}\text { Sign } \\
\left(\operatorname{det} J_{A E}\right)\end{array}$ & Critical eigenvalue(s) & Associated states \\
\hline $4 \cdot 4$ & + & + & $-0.0957 \pm j 10 \cdot 1407$ & $\delta_{2} \& \omega_{2}$ \\
\hline $4 \cdot 5$ & + & + & $0.0308 \pm \mathrm{j} 10.0034$ & $\delta_{2} \& \omega_{2}$ \\
\hline $4 \cdot 6$ & + & + & $0.3802 \pm j 9.9008$ & $\delta_{2} \& \omega_{2}$ \\
\hline $4 \cdot 7$ & + & + & $0.9344 \pm \mathrm{j} 10.1111$ & $\delta_{2} \& \omega_{2}$ \\
\hline $4 \cdot 8$ & + & + & $1 \cdot 3907 \pm j 11 \cdot 1963$ & $\delta_{2} \& \omega_{2}$ \\
\hline $4 \cdot 9$ & + & - & $24 \cdot 4174,0 \cdot 1104 \pm j 11 \cdot 3605$ & $E_{q 1}^{\prime} \& E_{f d 1}, \delta_{2} \& \omega_{2}$ \\
\hline $5 \cdot 0$ & + & - & 6.0978 & $E_{a 1}^{\prime} \& E_{f d 1}$ \\
\hline $5 \cdot 1$ & + & - & $2 \cdot 5680$ & $E_{a 1}^{\prime 1} \& E_{f d 1}$ \\
\hline $5 \cdot 15$ & + & - & 0.5417 & $E_{q 1}^{f+} \& E_{f d 1}$ \\
\hline 5.2 & \multicolumn{4}{|c|}{ Load flow does not converge } \\
\hline
\end{tabular}

Table 3. Modal behaviour of model $\mathrm{C}$ for different loads.

\begin{tabular}{|c|c|c|c|c|}
\hline $\begin{array}{l}\text { Load at } \\
\text { bus } 5\end{array}$ & $\begin{array}{c}\text { Sign } \\
\left(\operatorname{det} J_{L F}\right)\end{array}$ & $\begin{array}{c}\text { Sign } \\
\left(\operatorname{det} J_{A E}\right)\end{array}$ & Critical eigenvalue(s) & Associated states \\
\hline $4 \cdot 2$ & + & + & $-0.0048 \pm j 7.4848$ & $\delta_{2} \& \omega_{2}$ \\
\hline $4 \cdot 3$ & + & + & $0.2522 \pm j 7.4248$ & $\delta_{2} \& \omega_{2}$ \\
\hline $4 \cdot 4$ & + & + & $0.5333 \pm j 7.4024$ & $\delta_{2} \& \omega_{2}$ \\
\hline $4 \cdot 5$ & + & + & $0.8574 \pm j 7.4233$ & $\delta_{2} \& \omega_{2}$ \\
\hline $4 \cdot 6$ & + & + & $1.2592 \pm j 7.5151$ & $\delta_{2} \& \omega_{2}$ \\
\hline 47 & + & + & $1.8164 \pm j 7 \cdot 7697$ & $\delta_{2} \& \omega_{2}$ \\
\hline $4 \cdot 8$ & + & + & $2 \cdot 7800 \pm j 8 \cdot 6826$ & $\delta_{2} \& \omega_{2}$ \\
\hline 4.9 & + & - & $12 \cdot 2699,0.4398 \pm \mathrm{j} 10.0051$ & $E_{q 1}^{\prime} \& E_{f d 1}, \delta_{2} \& \omega_{2}$ \\
\hline $5 \cdot 0$ & + & - & $4 \cdot 1693,0 \cdot 1100 \pm j 9 \cdot 3208$ & $E_{a 1}^{q 1} \& E_{f d 1}^{\prime}, \delta_{2}^{2} \& \omega_{2}^{2}$ \\
\hline $5 \cdot 1$ & + & - & 1.6687 & $E_{a 1}^{\prime \prime} \& E_{\delta d 1}$ \\
\hline $5 \cdot 15$ & + & - & 0.0369 & $\delta_{1}^{q 1} \& \delta_{2}{ }^{\delta d 1}$ \\
\hline 5.2 & \multicolumn{4}{|c|}{ Load flow does not converge } \\
\hline
\end{tabular}

Table 4. Modal behaviour of model D for different loads.

\begin{tabular}{lcccc}
\hline Load at bus 5 & Sign (det $J_{L F}$ ) & Sign $\left(\right.$ det $J_{A E}$ ) & Critical eigenvalue(s) & Associated states \\
\hline 4.4 & + & + & $-0.2388 \pm \mathrm{j} 1.6434$ & $E_{q 1}^{\prime} \& R_{f 1}$ \\
4.5 & + & + & $-0.1997 \pm \mathrm{j} 1.7778$ & $E_{q 1}^{\prime} \& R_{f 1}$ \\
4.6 & + & + & $-0.1265 \pm \mathrm{j} 1.9985$ & $E_{q 1}^{\prime} \& R_{f 1}$ \\
4.7 & + & + & $0.0614 \pm \mathrm{j} 2.4531$ & $E_{q 1}^{\prime} \& R_{f 1}$ \\
4.8 & + & + & $1.7612 \pm \mathrm{j3} .9016$ & $E_{q 1}^{\prime} \& R_{f 1}$ \\
4.9 & + & - & 1.8483 & $E_{q 1}^{\prime} \& R_{f 1}$ \\
5.0 & + & - & 0.9059 & $E_{q 1}^{\&} \& R_{f 1}$ \\
5.1 & + & - & 0.3726 & $E_{q 1}^{\prime} \& R_{f 1}$ \\
$5 \cdot 15$ & + & - & -0.0424 & $E_{q 1}^{\prime}, \delta_{1} \& R_{f 2}$ \\
5.2 & & Load flow does not converge \\
\hline
\end{tabular}


bus $i$ is given by

$$
\begin{aligned}
& P_{L i}=P_{L i o}\left(V_{i} / V_{i o}\right)^{n_{p i}}, \quad i=1,2, \ldots, n, \\
& Q_{L i}=Q_{L i o}\left(V_{i} / V_{i o}\right)^{n_{q i} i}, \quad i=1,2, \ldots, n,
\end{aligned}
$$

where $P_{L i o}$ and $Q_{L i o}$ are the nominal real and reactive powers, respectively, at bus $i$, and $n_{p i}$ and $n_{q i}$ are the load indices. Three types of load werc considered.

(a) Constant power-type $\left(n_{p}=n_{q}=0\right)$;

(b) constant current-type $\left(n_{n}=n_{q}=1\right)$;

(c) constant impedance-type $\left(n_{p}=n_{q}=2\right)$.

Linearizing equations (62) and (63) and writing in the matrix form as in Padiyar et al (1986) we have

$$
\left[\begin{array}{l}
\Delta P_{L i} \\
\Delta Q_{L i}
\end{array}\right]=\left[\begin{array}{cc}
0 & n_{p i} \frac{P_{L i o}}{V_{i o}} \\
0 & n_{q i} \frac{Q_{L i o}}{V_{i o}}
\end{array}\right]\left[\begin{array}{l}
\Delta \theta_{i} \\
\Delta V_{i}
\end{array}\right], \quad i=1,2, \ldots, n
$$

Rewriting,

$$
\Delta S_{L i}=H_{i} \Delta V_{i}, \quad i=1,2, \ldots, n .
$$

In matrix form we get

$$
\Delta S_{L}=H \Delta V
$$

where $H$ is block diagonal. From (66), (60), and (61) we obtain,

$$
\Delta \dot{x}=\left[A+B H[I-D H]^{-1} C\right] \Delta x+E \Delta U .
$$

The step-by-step procedure of analysis for a given generating unit model is as follows:

(1) Select the type of the load at various buses (i.e., choose values of $n_{p}$ and $n_{q}$ );

(2) compute the system matrix

$$
A_{s y s}=A+B H[I-D H]^{-1} C \text {. }
$$

(3) Compute the sign of det $J_{L F}$, det $J_{A E}$ and the eigenvalues of $A_{s y s}$ for stability analysis.

For the three types of load mentioned earlier, the eigenvalues for the increased values of load are listed. For a nominal operating point of $P_{10}=1.5$ p.u., $Q_{10}=0.5$ p.u. at bus 5 , the system is dynamically stable for all three types of load. For an increased value of load at bus $5\left(P_{10}=4 \cdot 5, Q_{10}=0.5\right)$, the eigenvalues for model $\mathrm{A}$ are listed in table 5 . We observe that the system becomes dynamically unstable if the load is treated as a constant power type, whereas for the other two types of loads the system remains stable. Finally, we take another case $\left(P_{l o}=4.8, Q_{l o}=0.5\right)$, in which we show that the constant impedance type load is more stable than the constant current type (table 6 ). To demonstrate this condition we take model $B$ with a high gain of the exciter $\left(K_{A}=175\right)$. In this case the electromechanical mode becomes unstable. 
Table 5. Eigenvalues for different types of load at bus 5 for model A ( $P_{10}=4.5$ $\mathrm{pu}, Q_{l o}=0.5 \mathrm{pu}$ ).

\begin{tabular}{lll}
\hline $\begin{array}{l}\text { Constant power } \\
\text { (a) }\end{array}$ & $\begin{array}{c}\text { Constant current } \\
\text { (b) }\end{array}$ & $\begin{array}{c}\text { Constant impedance } \\
\text { (c) }\end{array}$ \\
\hline$-1.3774 \pm \mathrm{j} 12.8826$ & $-1.3439 \pm \mathrm{j} 12.9589$ & $-1.3387 \pm \mathrm{j} 12.9747$ \\
$-5.0224 \pm \mathrm{j} 9.5252$ & $-5.2555 \pm \mathrm{j} 9.7721$ & $-5.4441 \pm \mathrm{j} 9.8957$ \\
$-5.4233 \pm \mathrm{j} 9.9000$ & $-5.4366 \pm \mathrm{j} 9.8959$ & $-5.2854 \pm \mathrm{j} 9.8138$ \\
$-5.3342 \pm \mathrm{j} 9.8772$ & $-5.3355 \pm \mathrm{j} 9.8773$ & $-5.3366 \pm \mathrm{j} 9.8774$ \\
$-1.3923 \pm \mathrm{j} 6.8952$ & $-0.5359 \pm \mathrm{j} 8.0422$ & $-0.4857 \pm \mathrm{j} 8.2200$ \\
-5.5770 & -5.6721 & -5.7009 \\
-3.6361 & -3.8907 & -4.0346 \\
$0.3400 \pm \mathrm{j} 2.5538$ & $-0.4141 \pm \mathrm{j} 1.2391$ & $-0.4322 \pm \mathrm{j} 1.0369$ \\
-2.4979 & -3.3295 & -3.4099 \\
$-0.3876 \pm j 0.7800$ & $-0.3865 \pm \mathrm{j} 0.7865$ & $-0.3842 \pm \mathrm{j} 0.7929$ \\
$-0.4189 \pm \mathrm{j} 0.5827$ & $-0.4188 \pm \mathrm{j} 0.5825$ & $-0.4187 \pm \mathrm{j} 0.5824$ \\
-0.1973 & -0.1975 & -0.1976 \\
\hline
\end{tabular}

Table 6. Eigenvalues for different types of load at bus 5 for model $B\left(P_{10}=4.8\right.$ $\left.\mathrm{pu}, Q_{l o}=0.5 \mathrm{pu}\right), K_{A}=175$.

\begin{tabular}{|c|c|c|}
\hline $\begin{array}{l}\text { Constant power } \\
\text { (a) }\end{array}$ & $\begin{array}{l}\text { Constant current } \\
\text { (b) }\end{array}$ & $\begin{array}{l}\text { Constant impedance } \\
\text { (c) }\end{array}$ \\
\hline $\begin{aligned}-0.7671 \pm j 27.7009 \\
-0.0857 \pm j 17.1574 \\
0.5862 \pm j 9.9583 \\
-3.2680 \pm j 8.4323 \\
-2.8172 \pm j 7.4063 \\
-0.1988\end{aligned}$ & $\begin{array}{r}-0.1399 \pm j 17.0031 \\
-2.1787 \pm j 12.3851 \\
0.1244 \pm j 9.8034 \\
-3.1983 \pm j 8.3024 \\
-2.8136 \pm j 7.3841 \\
-0.1990\end{array}$ & $\begin{array}{l}-0 \cdot 1440 \pm j 17.0249 \\
-0 \cdot 1273 \pm j 10 \cdot 0391 \\
-2 \cdot 1013 \pm j 10 \cdot 7932 \\
-3 \cdot 1191 \pm j 8 \cdot 2135 \\
-2 \cdot 8089 \pm j 7.3650 \\
-0 \cdot 1991\end{array}$ \\
\hline
\end{tabular}

Table 7. Participation factors for various states of model A for the loading at bus $5=4.5 \mathrm{pu}$.

\begin{tabular}{|c|c|c|c|c|c|c|c|}
\hline \multirow{2}{*}{$\begin{array}{l}\text { Load at } \\
\text { bus } 5\end{array}$} & \multirow{2}{*}{$\begin{array}{l}\text { Unstable } \\
\text { cigenvalue(s) }\end{array}$} & \multicolumn{2}{|c|}{ Machine 1} & \multicolumn{2}{|c|}{ Machine 2} & \multicolumn{2}{|c|}{ Machine 3} \\
\hline & & State & $\mathrm{PF}$ & State & $\mathrm{PF}$ & State & PF \\
\hline \multirow{2}{*}{$4.5 \mathrm{pu}$} & \multirow{2}{*}{$0.3400 \pm j 2.5538$} & $\mathbf{E}_{q}^{\prime}$ & $0 \cdot 1984$ & $\mathrm{E}_{q}^{\prime}$ & $0 \cdot 1153$ & $E_{q}^{\prime}$ & 0.0768 \\
\hline & & $\mathbf{R}_{\boldsymbol{f}}$ & 0.1172 & $\begin{array}{l}E_{d}^{\prime} \\
R_{f}\end{array}$ & $\begin{array}{l}0.0853 \\
0.0641\end{array}$ & $E_{d}^{\prime}$ & 0.0603 \\
\hline
\end{tabular}

Table 8. Participation factors for various states of model $\mathrm{B}$ for the loading at bus $5=4 \cdot 5 \mathrm{pu}$.

\begin{tabular}{|c|c|c|c|c|c|c|c|}
\hline \multirow{2}{*}{$\begin{array}{l}\text { Load at } \\
\text { bus } 5\end{array}$} & \multirow{2}{*}{$\begin{array}{l}\text { Unstable } \\
\text { eigenvalue(s) }\end{array}$} & \multicolumn{2}{|c|}{ Machine 1} & \multicolumn{2}{|c|}{ Machine 2} & \multicolumn{2}{|c|}{ Machine 3} \\
\hline & & State & $\mathrm{PF}$ & State & $\mathrm{PF}$ & State & $\mathrm{PF}$ \\
\hline $4.5 \mathrm{pu}$ & $0.0308 \pm \mathrm{j} 10.0034$ & $\begin{array}{l}\delta \\
\omega\end{array}$ & $\begin{array}{l}0.0711 \\
0.0711\end{array}$ & $\begin{array}{c}\delta \\
\omega\end{array}$ & $\begin{array}{l}0.2724 \\
0.2724\end{array}$ & $\begin{array}{c}\delta \\
\omega\end{array}$ & $\begin{array}{l}0.0797 \\
0.0797\end{array}$ \\
\hline
\end{tabular}




\section{State variable participation in steady state stability and voltage collapse}

The participation factor analysis carried out leads to the following observations. Some of these agree with the results of Rajagopalan et al (1992).

The participation factors (PF) for a particular value of loading at bus 5 have been listed in tables 7 and 8 . Values below 0.05 have been neglected. The significant state variables are bold-face type.

Participation factor analyses of the eigenvalues for various cases reveal the following facts. For model $\mathrm{A}$, when the load is increased, it is observed that the critical modes for the unstable eigenvalues are the electrical ones $\left(E_{q}^{\prime}\right.$ and $\left.R_{f}\right)$. From table 1 we observe that when the load at bus 5 is increased from 4.7 pu to 4.8 pu, the complex pair of unstable eigenvalues splits into real ones which move in the opposite directions along the real axis. The one moving along the positive real axis $(9 \cdot 2464)$ is sensitive to the rotor angle mode and eventually comes back to the left-half plane via $+\infty$ when the load at bus 5 is increased from $4.8 \mathrm{pu}$ to $4.9 \mathrm{pu}$. This is the point when det $J_{A E}$ changes sign. The other unstable real eigenvalue moves to the left and is sensitive to the exciter mode. This eigenvalue returns to the left-half plane at the loading of approximately $5 \cdot 15$ pu at bus 5 , and the system is again dynamically stable. For the load at bus $5=5.2 \mathrm{pu}$, the load flow does not converge. It is possible through other algebraic techniques (Alvarado \& Jung 1989; Dobson \& Chiang 1989; Ajjarapu \& Christy 1992; Dobson \& Lu 1992) to reach the nose of the $P-V$ curve or the saddle node bifurcation. This phenomenon is pictorially indicated in the $P-V$ curve for model $\mathrm{A}$ and also in the trajectory of critical eigenvalue(s) in the s-plane (figures 7 and 8 ).

Between points $A$ and $B$ there is Hopf-bifurcation which has been shown to be subcritical. However load-flow solution still exists. In this region $E_{q}^{\prime}$ and $R_{f}$ state variables àre clearly dominant initially. As we approach $\mathrm{B}, \delta_{2}$ and $\omega_{2}$ state variables start participating substantially in the tiajectory of unstable eigenvalues as indicated in table 1 . For model $\mathrm{B}$ and model $\mathrm{C}$, which employ the fast static exciter with single time constant, the modes which go unstable are the electromechanical ones. For model $\mathrm{D}$, which again has an IEEE-type 1 exciter, the unstable eigenvalues correspond to the exciter variables. It is apparent that the modes which go unstable are critically dependent on the modelling of the exciter.

Hopf-bifurcation phenomena in power systems were first discussed by Abed \& Varaiya (1984) for a single machine case. In their studies the electro-mechanical mode

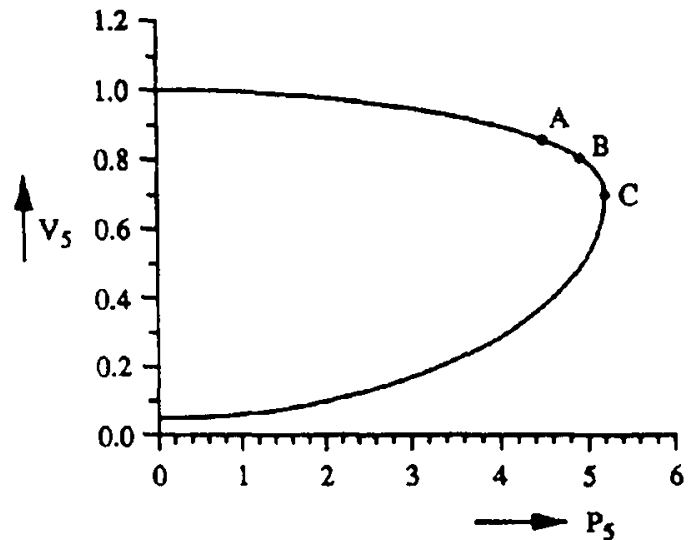

Figure 7. $P-V$ curve for bus 5 .

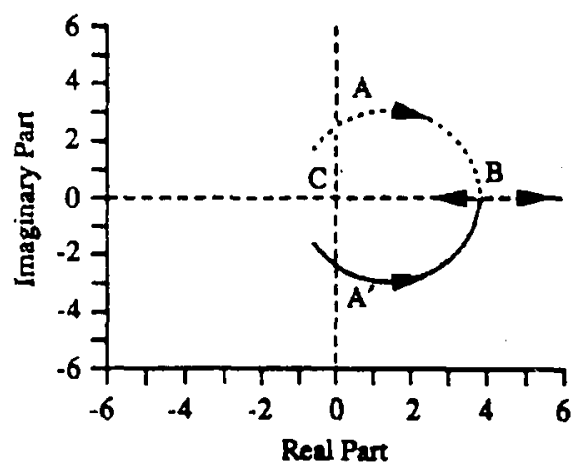

Figure 8. Critical modes of $\tilde{A}$ as a function of the load at bus 5 . 
was the critical one. In studies relating to voltage collapse (Rajagopalan et al 1992) that both exciter modes and electromechanical modes are critical in steady-state stability and voltage collapse and that they both participate in the dynamic instability. Hence decoupling the $Q-V$ dynamics from the $P-\delta$ dynamics as suggested by (Venkatasubramanian et al 1991) may not always hold. It may be true for special system configuration/operating conditions. There is no doubt that there are some underlying dynamics such as the load dynamics as discussed in Pal.(1992) and if such dynamics were represented, it will be fast dynamics and the phenomena of det $J_{A E}$ changing sign will still exist. In conventional bifurcation theory terms one can think of solving $g(x, y)=0$ for $y=h(x)$ and substituting this in the differential equation to get $\dot{x}=f(x, h(x))$. The change in sign of det $J_{A E}$ is the instant when solution of $y$ is no longer possible. This is also tied in with the concept of implicit function theorem in singular perturbation theory. A concrete mathematical underpinning of these ideas in the context of power systems is a research issue. Recent work in "singularly induced bifurcation" (Venkatasubramanian et al 1992) and "impasse surface" concepts (Hiskens \& Hill 1991) may throw more light.

\section{Conclusion}

In this paper we have developed a comprehensive linear model for both steady-state and voltage-stability analyses including nonlinear voltage-dependent loads. A parametric analysis has been done and use of participation factor is particularly relevant. Further research is needed to include load tap changer, FACTS dynamics and load dynamics.

This research work was supported in part by EPRI through its project EPRI 8010-21 and the National Science Foundation through its grant ECS 91-19428.

\section{References}

Abed E H, Varaiya P P 1984 Nonlinear oscillations in power systems. Int. J. Electr. Power Energy Syst. 6: 37-43

Ajjarapu V, Christy C 1992 The continuation power flow: a tool for steady-state voltage stability analysis. IEEE Trans. Power Syst. 1: 416-423

Alvarado F L, Jung T H 1989 Direct detection of voltage collapse conditions. Proceedings: Bulk Power System Voltage Phenomena - Voltage Stability and Security (Palo Alto, CA:EPRI)

Anderson P M, Fouad A A 1977 Power system control and stability (Ames, IA: Iowa State University Press)

Dobson I, Chiang H D 1989 Towards a theory of voltage collapse in electric power systems. Syst. Control Lett. 13: 253-262

Dobson I, Lu L 1992 New methods for computing a closest saddle node bifurcation and worst case load power margin for voltage collapse. IEEE Summer Power Meeting, Paper 92 SM 587-6 PWRS

Hiskens I A, Hill D J 1991 Failure modes of a collapsing power system. Proc. Bulk Power System Voltage Phenomena II - Voltage Stability and Security (Fairfax, VA:ECC Inc.) 
Kundur P, Rogers G J, Wong D Y, Wang L, Lauby M G 1990 A comprehensive computer program package for small signal stability analysis of power systems. JEEE Trans. Power Syst. 5: 1076-1083

Padiyar K R, Rajasekharam P, Radhakrishna C, Pai M A 1986 Dynamic stabilization of power systems through reactive power modulation. Electr. Mach. Power Syst. 11:281-293

Padiyar K R, Pai M A, Radhakrishna C 1981 A versatile system model for the dynamic stability analysis of power systems including HVDC links. IEEE Trans. Power Appar. Syst. 100: $1871-1880$

Pal M K 1992 Voltage stability conditions considering load characteristics. IEEE Trans. Power Syst. 7: 243-249

Rajagopalan C, Lesieutre B, Sauer P W, Pai M A 1992 Dynamic aspects of voltage/power characteristics. IEEE Trans. Power Syst. 7: 990-1000

Sauer P W, Pai M A 1990 Power system steady-state stability and the load flow Jacobian. IEEE Trans. Power Syst. 5: 1374-1383

Sauer P W, Pai M A 1991 Modelling and simulation of multi-machine power system dynamics. Control and dynamic systems: Advances in theory and application (ed.) C T Leondes (San Diego, CA: Academic Press) vol. 43

Venkatasubramanian V, Schattler H, Zaborsky J 1991 A taxonomy of the dynamics of a large power system with emphasis on its voltage stability. Proc. Bulk Power System Voltage Phenomena II - Voltage Stability and Security (Fairfax, VA:ECC Inc.)

Venkatasubramaniam V, Schattler H, Zaborsky J 1992 Voltage dynamics: study of a generator with voltage control, transmission and matched mw load. IEEE Trans. Autom. Control. 37: $1717-1733$

Verghese G C, Perez-Arriaga IJ, Scheweppe F C 1982 Selective modal analysis with applications to electric power systems, Parts I and II. IEEE Trans. Power Appar. Syst. PAS-101: $3117-3134$

Wang L, Semlyen A 1990 Application of sparse eigenvalue techniques to the small signal stability analysis of large power systems. IEEE Trans. Power Syst. 5: 635-642 